\title{
Factorization of gravitational Compton scattering amplitude in the linearized version of general relativity
}

\author{
S. Y. Choi, J. S. Shim, and H. S. Song \\ Center for Theoretical Physics and Department of Physics, \\ Seoul National University, Seoul 151-742, Korea
}

\begin{abstract}
Gravitational Compton scattering process with a massive fermion is studied in the context of the linearized gravity. Gravitational gauge invariance and graviton transversality cause the transition amplitude to be factorized into that of scalar QED Compton scattering and that of fermion QED Compton scattering with an overall kinematical factor. The factorization is shown explicitly and its physical implications are discussed.
\end{abstract}

Pacs numbers : 12.25.+e, 04.60.+n, 13.88.+e

Typeset using REVTEX 
Newton's long-ranged gravity force law suggests the force to be mediated by a massless particle called a graviton and several observations of universal gravitational attraction support a spin-2 graviton picture [1].

Until now, there is no complete theory of quantum gravity. One of its main problems is that Einstein gravity is nonrenormalizable [2] because the gravitational constant $G_{N}$ has dimensions of inverse mass square. This aspect implies that the Einstein theory of gravitation seems to be more like other nonrenormalizable effective theories such as the Fermi theory of weak interaction. Incidentally, Weinberg [3] showed that it is impossible to construct a Lorentz-invariant quantum theory of particles of mass zero and helicity \pm 2 without introducing some sort of gauge invariance into the theory. On the other hand, even if it is nonrenormalizable, the classical theory of gravitational radiation in the linearized version of general relativity is well known to have gauge invariance related to the general covariance of the full theory. The fact uniquely determines the long wavelength structure of quantum gravitation to be that of Einstein's theory [3, [4].

Several graviton interaction processes have been studied previously. The cross section of tree-level gravitational Compton scattering $g e \rightarrow g e$ was calculated in Ref. [5]. The cross section of an annihilation process $e \bar{e} \rightarrow g g$ was calculated some time ago in Ref. [6]. However, none of the two calculations were consistent with the result of Voronov [7], whose Lagrangian is gravitationally gauge invariant. Whatever the theory is, it is so crucial to maintain general covariance in the theory that one has to introduce gravitational gauge invariance in the interaction Lagrangian of linearized gravity [3]. The fact justifies the use of the same interaction Lagrangian as in Ref. [7].

Gravitational gauge invariance and transversality of graviton tensors cause the amplitude of gravitational Compton scattering to be completely factorized into that of scalar QED Compton scattering and that of fermion QED Compton scattering, both of which are gauge invariant. One overall kinematical factor clearly exhibits the unitarity violation of the process at quite high energies. In this report, we show the factorization explicitly and discuss its physical implications 
The Lagrangian $\mathcal{L}$ []] of the interaction of a massive fermion with the gravitational field has two terms : the Lagrangian $\mathcal{L}_{g}$ of the pure gravitational field and the general covariant Lagrangian $\mathcal{L}_{f}$ of the massive fermion

$$
\begin{aligned}
\mathcal{L} & =\mathcal{L}_{g}+\mathcal{L}_{f} \\
\mathcal{L}_{g} & =\frac{1}{\kappa^{2}} \sqrt{-g} g^{\mu \nu}\left[\Gamma_{\mu \nu}{ }^{\alpha} \Gamma_{\alpha \beta}{ }^{\beta}-\Gamma_{\mu \alpha}{ }^{\beta} \Gamma_{\nu \beta}{ }^{\alpha}\right] \\
\mathcal{L}_{f} & =\sqrt{-g}\left[\frac{i}{2}\left(\bar{\psi} \gamma^{i} \nabla_{i} \psi-\tilde{\nabla}_{i} \bar{\psi} \gamma^{i} \psi\right)-m \bar{\psi} \psi\right] .
\end{aligned}
$$

where $g=\operatorname{det}\left[g_{\mu \nu}\right], \kappa=\sqrt{16 \pi G_{N}}, \gamma^{i}=\lambda_{\mu}^{i} \gamma^{\mu}$ with a certain reference $\lambda_{\mu}^{i}$ and ordinary Dirac matrices $\gamma^{\mu}$, and

$$
\nabla_{i} \psi=\psi_{, i}-\Gamma_{i} \psi, \quad \tilde{\nabla}_{i} \bar{\psi}=\bar{\psi}_{, i}+\bar{\psi} \Gamma_{i}
$$

where $\Gamma_{i}=\gamma^{\mu} \gamma^{\nu} \lambda_{\mu, i}^{k} \lambda_{\nu k}$. With general covariance preserved, it is convenient to choose the reference uniquely connected with the metric tensor. The choice of reference in Ref. [8] is

$$
\lambda_{\mu i}=\sqrt{g_{\mu i}}
$$

with the square taken in the matrix sense.

Let us consider a symmetric and traceless tensor $h_{\mu \nu}$ representing a deviation of the metric tensor $g_{\mu \nu}$ from the flat tensor $\eta_{\mu \nu}$ :

$$
g_{\mu \nu}=\eta_{\mu \nu}+\kappa h_{\mu \nu}
$$

where the flat tensor $\eta_{\mu \nu}$ is the Minkowski tensor with signature $(+,-,-,-)$. Expanding the Lagrangian $\mathcal{L}_{g}$ up to the second order of $\kappa$, we have

$$
\begin{aligned}
\mathcal{L}_{g} & =\mathcal{L}_{g}^{0}+\kappa \mathcal{L}_{g}^{1}+\kappa^{2} \mathcal{L}_{g}^{2}, \\
\mathcal{L}_{g}^{0} & =-\frac{1}{4}\left[2 \partial_{\alpha} h_{\mu \nu} \partial^{\nu} h^{\mu \alpha}-\partial_{\alpha} h \partial_{\mu} h^{\mu \alpha}+\partial^{\mu} h \partial_{\mu} h-\partial_{\alpha} h^{\mu \nu} \partial^{\alpha} h_{\mu \nu}\right], \\
\mathcal{L}_{g}^{1} & =\frac{1}{4}\left[\left(h_{\mu \nu}-\frac{1}{2} \eta_{\mu \nu} h\right) \eta_{\alpha \gamma} \eta_{\beta \delta}+\eta_{\mu \nu} \eta_{\beta \delta} h_{\alpha \gamma}+\eta_{\mu \nu} \eta_{\alpha \gamma} h_{\beta \delta}\right] \\
& \times\left[2 \partial^{\beta} h^{\gamma \mu} \partial^{\alpha} h^{\delta \nu}-2 \partial^{\delta} h^{\beta \gamma} \partial^{\alpha} h^{\mu \nu}+\partial^{\nu} h^{\alpha \gamma} \partial^{\mu} h^{\beta \delta}-\partial^{\mu} h^{\beta \gamma} \partial^{\nu} h^{\alpha \delta}\right],
\end{aligned}
$$


and $\mathcal{L}_{g}^{2}$ is a certain complicated polynomial of fourth degree in $h_{\mu \nu}$ and $h=\eta^{\mu \nu} h_{\mu \nu}$. The full expression of $\mathcal{L}_{g}^{2}$ can be found in Ref. [9]. For the general covariant Lagrangian of the massive fermion we have in an analogous manner

$$
\begin{aligned}
\mathcal{L}_{f} & =\mathcal{L}_{f}^{0}+\kappa \mathcal{L}_{f}^{1}+\kappa^{2} \mathcal{L}_{f}^{2} \\
\mathcal{L}_{f}^{0} & =\frac{i}{2}\left[\bar{\psi} \gamma^{\mu} \partial_{\mu} \psi-\partial_{\mu} \bar{\psi} \gamma^{\mu} \psi\right]-m \bar{\psi} \psi \\
\mathcal{L}_{f}^{1} & =\frac{1}{2} h \mathcal{L}_{f}^{0}-\frac{i}{4} h_{\mu \nu}\left[\bar{\psi} \gamma^{\nu} \partial^{\mu} \psi-\partial^{\mu} \bar{\psi} \gamma^{\nu} \psi\right] \\
\mathcal{L}_{f}^{2} & =\frac{1}{8}\left(h^{2}-2 h^{\mu \nu} h_{\mu \nu}\right) \mathcal{L}_{f}^{0}+\frac{i}{16}\left(3 h_{\mu \alpha} h_{\nu}^{\alpha}-2 h h_{\mu \nu}\right)\left(\bar{\psi} \gamma^{\mu} \partial^{\nu} \psi-\partial^{\nu} \bar{\psi} \gamma^{\mu} \psi\right) \\
& +\frac{i}{32}\left[2\left(h_{\mu \alpha} \partial^{\alpha} h^{\mu \nu}-h^{\mu \nu} \partial^{\alpha} h_{\mu \alpha}\right)\left(\bar{\psi} \gamma_{\nu} \psi\right)+\left(h^{\mu \nu} \partial^{\alpha} h_{\mu}^{\beta}-h^{\beta \mu} \partial^{\alpha} h_{\mu}^{\nu}\right)\left(\bar{\psi} \gamma_{\alpha} \gamma_{\beta} \gamma_{\gamma} \psi\right)\right] .
\end{aligned}
$$

The gravitational Compton scattering process $g e \rightarrow g e$ is made up of four Feynman diagrams (See Fig. 1) and the explicit form of its tree-level transition amplitude $\mathcal{M}_{g g}$ is determined from the Lagrangian $\mathcal{L}$ as

$$
\begin{aligned}
& \mathcal{M}_{g g}=\mathcal{M}_{a}+\mathcal{M}_{b}+\mathcal{M}_{c}+\mathcal{M}_{d} \\
& \mathcal{M}_{a}=-\frac{\kappa^{2}}{4} \frac{\left(p_{1} \cdot \epsilon_{1}\right)\left(p_{2} \cdot \epsilon_{2}^{*}\right)}{\left(p_{1} \cdot k_{1}\right)} \bar{u}\left(p_{2}\right)\left[\phi_{2}^{*}\left(\not p_{1}+\not \ell_{1}+m\right) \phi_{1}\right] u\left(p_{1}\right), \\
& \mathcal{M}_{b}=\frac{\kappa^{2}}{4} \frac{\left(p_{2} \cdot \epsilon_{1}\right)\left(p_{1} \cdot \epsilon_{2}^{*}\right)}{\left(p_{1} \cdot k_{2}\right)} \bar{u}\left(p_{2}\right)\left[\notin_{1}\left(\not p_{1}-\not k_{2}+m\right) k_{2}^{*}\right] u\left(p_{1}\right), \\
& \mathcal{M}_{c}=\frac{\kappa^{2}}{2\left(k_{1} \cdot k_{2}\right)}\left[\left(p_{2} \cdot \epsilon_{2}^{*}\right)\left(p_{1} \cdot \epsilon_{1}\right)-\left(p_{1} \cdot \epsilon_{2}^{*}\right)\left(p_{2} \cdot \epsilon_{1}\right)-\left(p_{1} \cdot k_{1}\right)\left(\epsilon_{1} \cdot \epsilon_{2}^{*}\right)\right] \\
& \times \bar{u}\left(p_{2}\right)\left[\phi_{1}\left(k_{1} \cdot \epsilon_{2}^{*}\right)-\not k_{1}\left(\epsilon_{1} \cdot \epsilon_{2}^{*}\right)+\not \phi_{2}^{*}\left(k_{2} \cdot \epsilon_{1}\right)\right] u\left(p_{1}\right) \\
& -\frac{\kappa^{2}}{2}\left[\epsilon_{1} \cdot \epsilon_{2}^{*}\right] \bar{u}\left(p_{2}\right)\left[\phi_{1}\left(p_{1} \cdot \epsilon_{2}^{*}\right)+\phi_{2}^{*}\left(p_{2} \cdot \epsilon_{1}\right)+\frac{\not \kappa_{1}}{2}\left(\epsilon_{1} \cdot \epsilon_{2}^{*}\right)\right] u\left(p_{1}\right), \\
& \mathcal{M}_{d}=\frac{\kappa^{2}}{2}\left[\epsilon_{1} \cdot \epsilon_{2}^{*}\right] \bar{u}\left(p_{2}\right)\left[\frac{1}{2} \phi_{1}\left(\not p_{1}-\not k_{2}+m\right) \notin_{2}^{*}+\phi_{1}\left(p_{2} \cdot \epsilon_{2}^{*}\right)+\notin_{2}^{*}\left(p_{1} \cdot \epsilon_{1}\right)-\frac{\not k_{1}}{2}\left(\epsilon_{1} \cdot \epsilon_{2}^{*}\right)\right] u\left(p_{1}\right) \text {, }
\end{aligned}
$$

where $\epsilon_{1}^{\mu} \epsilon_{1}^{\nu}$ and $k_{1}^{\mu}$ are the initial polarization and momentum of the graviton, $\epsilon_{2}^{* \mu} \epsilon_{2}^{* \nu}$ and $k_{2}^{\mu}$ are the final polarization and momentum of the graviton, $p_{1}^{\mu}$ and $p_{2}^{\mu}$ are the initial and final momenta of the massive fermion. The transition amplitude $\mathcal{M}_{g g}$ is invariant under the gravitational gauge transformation

$$
\epsilon_{i}^{\mu} \rightarrow \epsilon_{i}^{\mu}+\Lambda_{i} k_{i}^{\mu}, \quad i=1,2
$$


where $\Lambda_{i}(i=1,2)$ are arbitrary scalar functions. The combination of two complicated $\mathcal{M}_{c}$ and $\mathcal{M}_{d}$ leads to a simple expression as

$$
\begin{aligned}
\mathcal{M}_{e} & =\frac{\kappa^{2}}{2\left(k_{1} \cdot k_{2}\right)}\left[\left(p_{2} \cdot \epsilon_{2}^{*}\right)\left(p_{1} \cdot \epsilon_{1}\right)-\left(p_{1} \cdot \epsilon_{2}^{*}\right)\left(p_{2} \cdot \epsilon_{1}\right)-\left(p_{1} \cdot k_{2}\right)\left(\epsilon_{1} \cdot \epsilon_{2}^{*}\right)\right] \\
& \times \bar{u}\left(p_{2}\right)\left[\phi_{1}\left(k_{1} \cdot \epsilon_{2}^{*}\right)-\not k_{1}\left(\epsilon_{1} \cdot \epsilon_{2}^{*}\right)+\phi_{2}^{*}\left(k_{2} \cdot \epsilon_{1}\right)\right] u\left(p_{1}\right) \\
& +\frac{\kappa^{2}}{4}\left[\epsilon_{1} \cdot \epsilon_{2}^{*}\right] \bar{u}\left(p_{2}\right)\left[\phi_{1}\left(\not p_{1}-\not k_{2}+m\right) \phi_{2}^{*}\right] u\left(p_{1}\right) .
\end{aligned}
$$

On the other hand, all three amplitude parts $\left(\mathcal{M}_{a}, \mathcal{M}_{b}\right.$ and $\left.\mathcal{M}_{e}\right)$ are not independent. Transversality of graviton polarization yields the relation,

$$
\begin{aligned}
\bar{u}\left(p_{2}\right)\left[\phi_{1}\left(k_{1} \cdot \epsilon_{2}^{*}\right)-\not k_{1}\left(\epsilon_{1} \cdot \epsilon_{2}^{*}\right)+\not \phi_{2}^{*}\left(k_{2} \cdot \epsilon_{1}\right)\right] u\left(p_{1}\right) \\
=\frac{1}{2} \bar{u}\left(p_{2}\right)\left[\phi_{2}^{*}\left(\not p_{1}+\not \ell_{1}+m\right) \not \phi_{1}-\not \phi_{1}\left(\not p_{1}-\not k_{2}+m\right) \phi_{2}^{*}\right] u\left(p_{1}\right),
\end{aligned}
$$

and Eq. (10) renders the transition amplitude $\mathcal{M}_{g g}$ completely factorized,

$$
\begin{aligned}
\mathcal{M}_{g g} & =-\frac{\kappa^{2}}{2} F\left[\left(\epsilon_{1} \cdot \epsilon_{2}^{*}\right)-\frac{\left(p_{1} \cdot \epsilon_{1}\right)\left(p_{2} \cdot \epsilon_{2}^{*}\right)}{\left(p_{1} \cdot k_{1}\right)}+\frac{\left(p_{2} \cdot \epsilon_{1}\right)\left(p_{1} \cdot \epsilon_{2}^{*}\right)}{\left(p_{1} \cdot k_{2}\right)}\right] \\
& \times \bar{u}\left(p_{2}\right)\left[\frac{\phi_{2}^{*}\left(\not p_{1}+\not k_{1}+m\right) \phi_{1}}{2\left(p_{1} \cdot k_{1}\right)}-\frac{\phi_{1}\left(\not p_{1}-\not k_{2}+m\right) \notin_{2}^{*}}{2\left(p_{1} \cdot k_{2}\right)}\right] u\left(p_{1}\right),
\end{aligned}
$$

where the overall kinematical factor $F$ is

$$
F=\frac{\left(p_{1} \cdot k_{1}\right)\left(p_{1} \cdot k_{2}\right)}{\left(k_{1} \cdot k_{2}\right)},
$$

and two bracket factors are nothing but the transition amplitudes of scalar and fermion QED Compton scattering processes, respectively. The conclusion is that the gravitational Compton scattering amplitude is the direct folding of scalar and fermion QED Compton scattering amplitudes with an overall kinematical factor $F$.

The factorization property is not only confined to the linearized gravity but it is quite general. As a matter of fact, any non-Abelian theory with a semi-simple gauge group has similar factorization properties [10]. For instance, the transition amplitude of a gluon scattering with a colored massive quark also is completely factorized into one part dependent only on the non-Abelian gauge group structure as well as kinematics and the other part 
which is nothing but the fermion QED Compton scattering amplitude. The group-dependent part takes a simple expression in terms of an anticommutator and a commutator of group generators $T^{a}$ and $T^{b}$ as

$$
\left\{T^{a}, T^{b}\right\}_{i j}-\frac{p_{1} \cdot\left(k_{1}+k_{2}\right)}{\left(k_{1} \cdot k_{2}\right)}\left[T^{a}, T^{b}\right]_{i j}
$$

where $i$ and $j$ are color indices for the initial and final fermions, $a$ and $b$ are gluon indices for the initial and final gluons, respectively. Moreover, the linearized gravity coupled with QED has the amplitude $\mathcal{M}_{\gamma g}$ of the graviton photoproduction $\gamma e \rightarrow$ ge factorized [1]

$$
\mathcal{M}_{\gamma g}=\frac{\kappa}{4 e}\left[n \cdot \epsilon_{g}^{*}\right] \mathcal{M}_{\gamma \gamma}
$$

where $e$ and $\mathcal{M}_{\gamma \gamma}$ are the electron charge and the QED Compton scattering amplitude, respectively, and

$$
n^{\mu}=\left[\left(p_{1}+p_{2}\right)^{\mu}-\frac{p_{1} \cdot\left(k_{1}+k_{2}\right)}{\left(k_{1} \cdot k_{2}\right)}\left(k_{1}+k_{2}\right)^{\mu}\right] .
$$

In brief, each amplitude of a gauge boson scattering with a massive fermion is completely factorized into two parts in any theory with unbroken gauge symmetries, where one spindependent part is of the same form as the well-known QED Compton scattering amplitude and the other is independent of the spin of matter fields. The independence of matter spin is still preserved in the linearized gravity. But, it is one unique characteristic of the gravitational Compton scattering that both amplitude factors of the process depend on graviton polarization, whereas one amplitude factor of any other process does not depend on gauge boson polarization.

Let us investigate the graviton polarization dependence of scalar QED Compton amplitude in Eq. (11) in more detail. Masslessness requires a graviton to have only two helicity states. One useful polarization basis [7, 12] consists of two four-vectors

$$
n_{1}^{\mu}=\frac{1}{2} N n^{\mu}, \quad n_{2}^{\mu}=N \frac{\varepsilon^{\mu \nu \alpha \beta} p_{1 \nu} k_{2 \alpha} k_{1 \beta}}{\left(k_{1} \cdot k_{2}\right)},
$$

with the normalization factor $N=1 / \sqrt{2 F-m^{2}}$. The vectors $n_{1}$ and $n_{2}$ satisfy the following relations: 


$$
\begin{aligned}
& n_{i} \cdot n_{j}=-\delta_{i j}, \quad p_{i} \cdot n_{2}=k_{j} \cdot n_{i}=0, \\
& p_{i} \cdot n_{1}=\left(\frac{s u-m^{2}}{t}\right)^{1 / 2}, \quad(i, j=1,2),
\end{aligned}
$$

where $s, t$, and $u$ are Mandelstam variables. The basis enables us to simultaneously construct the polarization vectors $\epsilon_{1}(\lambda)$ and $\epsilon_{2}^{*}(\lambda)(\lambda= \pm 1)$ for the initial and final gravitons as

$$
\epsilon_{1}(\lambda)=\epsilon_{2}^{*}(\lambda)=\frac{1}{\sqrt{2}}\left(n_{1}+i \lambda n_{2}\right)
$$

Any basis change gives rise to only an overall phase and so it gives no effect on any physical observable. In the amplitude, only the direct inner product $\left[\epsilon_{1} \cdot \epsilon_{2}^{*}\right]$ is dependent on graviton polarization:

$$
\epsilon_{i}(\lambda) \cdot \epsilon_{2}^{*}\left(\lambda^{\prime}\right)=-\delta_{\lambda,-\lambda^{\prime}}
$$

Conventional gauge theories do not show this polarization dependence and hence the polarization difference could be utilized for discriminating between graviton interactions and other gauge-boson interactions.

Both the dependence (19) on graviton polarization and the comparison of (15) with (13) imply that a four-momentum serves as a gravitational charge, while graviton helicities serve as gauge-group indices in the linearized gravity. On the other hand, the overall kinematical factor $F$ reads in the center of mass frame

$$
F=\frac{1}{2}\left[s \frac{1+\cos \theta}{1-\cos \theta}+m^{2}\right], \quad \theta=\text { angle between gravitons }
$$

and its linear $s$-dependence causes unitarity violation at high energies.

In conclusion, the factorization is a common property of every field theory with unbroken local symmetries. The factorization maintenance of Einstein gravity strengthens much more that the theory should be the very low-energy limit of the ultimate guantum gravitational theory [3,4]. While it might be at present beyond reach to take a great conceptual leap toward the ultimate gravitational theory, it looks clear that some crucial modifications must be considered in factors, which are independent of the spin of matter fields. 


\section{ACKNOWLEDGMENTS}

The work is supported in part by the Korea Science and Engineering Foundation through the SRC program and in part by the SNU Daewoo Research Fund. 


\section{REFERENCES}

[1] R. P. Feynman, Lectures on Gravitation, (Caltech Notes, Pasadena, 1962, unpublished); C. M. Will, in Three hundred years of gravitation, edited by S. W. Hawking and W. Israel (Cambridge University Press, Cambridge, England, 1987); C. W. Misner, K. S. Thorne, and J. A. Wheeler, Gravitation (Freeman, San Francisco, 1973), Part IX.

[2] S. Deser and P. van. Nieuwenhuizen, Phys. Rev. D 10, 401 (1974); 10, 411 (1974); B. S. DeWitt, Phys. Rev. 160, 1113 (1967); 162, 1195 (1967); 162, 1239 (1967); S. Weinberg, Garvitation and Cosmology: Principles and Applications of the General Theory of Relativity (Wiley, New York, 1972), Chap 10.

[3] S. Weinberg, Phys. Rev. 138, B988 (1965).

[4] D. G. Boulware and S. Deser, Ann. Phys. (N.Y.) 89, 193 (1975).

[5] S. N. Gupta, Proc. Phys. Soc., London, A65, 161 (1952); A65, 608 (1952); Phys. Rev. 96, 1683 (1954); V. N. Mironovskil, Zh. Eksp. Teor. Fiz. 48, 358 (1965) [Sov. Phys. JETP 21, 236 (1965)]; B. M. Barker, M. S. Bhatia and S. N. Gupta, ibid. 182, 1387 (1969).

[6] Yu. S. Vladimirov, Zh. Eksp. Teor. Fiz. 45, 251 (1963) [Sov. Phys. JETP 18, 178 (1964)].

[7] N. A. Voronov, Zh. Eksp. Theor. Fiz. 64, 1889 (1973) [Sov. Phys. JETP 37, 953 (1973)].

[8] V. I. Ogievetskii and I. V. Polubarinov, Zh. Eksp. Theor. Fiz. 48, 1625 (1965) [Sov. Phys. JETP 21, 1093 (1965)].

[9] D. Gross and R. Jackiw, Phys. Rev. 166, 1287 (1968).

[10] T. R. Grose and K. O. Mikaelian, Phys. Rev. D 23, 123 (1981); Z. Dongpei, Phys. Rev. D 22, 2266 (1980); C. J. Goebel, F. Halzen, and J. P. Leveille, Phys. Rev. D 23, 2682 (1981); S. J. Brodsky and R. W. Brown, Phys. Rev. Lett. 49, 966 (1982); R. W. Brown, K. L. Kowalski, and S. J. Brodsky, Phys. Rev. D 28, 624 (1983). 
[11] S. Y. Choi, Jungil Lee, J. S. Shim, and H. S. Song, to appear in Phys. Rev. D.

[12] V. B. Berestetskii, E. M. Lifshitz and L. P. Pitaevskii, Relativistic Quantum Theory (Pergamon, Oxford, 1979), Part I. 


\section{FIGURES}

FIG. 1. Feynman diagrams for the gravitational Compton scattering process. The curly line is for a graviton and the solid line for a massive fermion. 